Изв. Крымск. Астрофиз. Обсерв. 117, № 1, 38-43 (2021) doi:10.31059/izcrao-vol117-iss1-pp38-43

УДК 523.985

\title{
Предвестники солнечных вспышек в микроволновом диапазоне
}

\author{
B.Е. Абрамов-Максимов ${ }^{1}$, И.А. Бакунина ${ }^{2}$ \\ 1 Главная (Пулковская) астрономическая обсерватория РАН, Пулковское шоссе, 65/1, Санкт-Петербург, \\ 196140, Россия \\ beam@gaoran.ru \\ 2 Национальный исследовательский университет "Высшая школа экономики", ул. Б. Печерская, 25/12, \\ Нижний Новгород, 603155, Россия
}

Поступила в редакцию 31 октября 2021 г.

\begin{abstract}
Аннотация. Представлено исследование пространственного распределения квазипериодических колебаний (KПК) микроволнового излучения в двух активных областях перед вспышками М-класса. Мы рассмотрели два случая: NOAA 112836 сентября и NOAA 1130225 сентября 2011 г. Использовались ежедневные наблюдения на радиогелиографе Nobeyama (NoRH) на частоте 17 ГГц. В обоих случаях были обнаружены предвспышечные цуги колебаний яркостной температуры микроволнового излучения активных областей. Длительность цугов составляет около 3-4 циклов колебаний. В обоих случаях источником обнаруженных колебаний являлась компактная зона в активной области, которая совпадает с областью максимальной яркости во время вспышки.
\end{abstract}

Ключевые слова: Солнце, вспышки, радиоизлучение, квазипериодические колебания

\section{1 Введение}

Квазипериодические колебания (КПК), или флуктуации (КПФ), микроволнового излучения солнечных активных областей (AO) были открыты около 50 лет назад (Durasova et al., 1971). Практически сразу же были получены указания на наличие связи между параметрами КПК и вспышечной активностью (Кобрин и др., 1973a, b; Алешин и др., 1973; Коршунов, Прокофьева, 1976). Независимо разными авторами был обнаружен эффект усиления мощности КПК микроволнового излучения непосредственно перед вспышкой (Берулис и др., 1973; Арбузов и др., 1979; Кобрин и др, 1981; Аверьянихина и др., 1982; Берулис и др., 1983; Авдюшин и др., 1985). Более того, был обнаружен аналогичный эффект усиления пульсаций геомагнитного поля перед протонными вспышками и его связь с пульсациями радиоизлучения Солнца (Быстров и др., 1978, 1979).

Однако возможности инструментов 70-80-х годов были довольно ограниченны. Значительное продвижение в исследованиях КПК в микроволновом диапазоне произошло благодаря созданию радиогелиографа Nobeyama (NoRH) (Gelfreikh et al., 1999, 2006). Ha NoRH проводились непрерывные наблюдения Солнца с 1992 по 2020 г. по 7-8 ч ежедневно с временным разрешением 1 с в штатном режиме на частотах 17 и 34 ГГц с регистрацией интенсивности (параметр Стокса I) и круговой поляризации (параметр Стокса V, только на 17 ГГц). Угловое разрешение составляет 10-20" на частоте 17 ГГц, что позволяет выделять в АО отдельные компоненты радиоизлучения. С использованием данных наблюдений NoRH был обнаружен ряд случаев возрастания мощности КПК перед вспышками с периодами от 3 до 100 минут (Sych et al., 2009; Abramov-Maximov et al., 2011; АбрамовМаксимов, Бакунина, 2018; Abramov-Maximov, Bakunina, 2019, 2020). Подобный эффект усиления KПК перед вспышками был обнаружен в мягком рентгеновском излучении по данным GOES (Tan et al., 2016).

Во время вспышки происходит существенная перестройка магнитного поля АО и, как следствие, резкое изменение параметров плазмы, что, вероятно, и приводит к изменению наблюдаемых характеристик КПК. Это может быть использовано для диагностики корональной плазмы. С одной 
стороны, анализ предвспышечных КПК излучения АО может помочь продвинуться в понимании процессов, происходящих при развитии вспышки, а с другой стороны, возможно, разработать новые краткосрочные критерии прогноза геоэффективных вспышек.

Целью данной работы является исследование пространственного распределения КПК, наблюдаемых перед вспышками в активных областях

\section{2 Наблюдения}

\section{1 Используемые данные наблюдений и метод обработки}

NOAA 11283 2011-09-06 NoRH $17 \mathrm{GHz}$

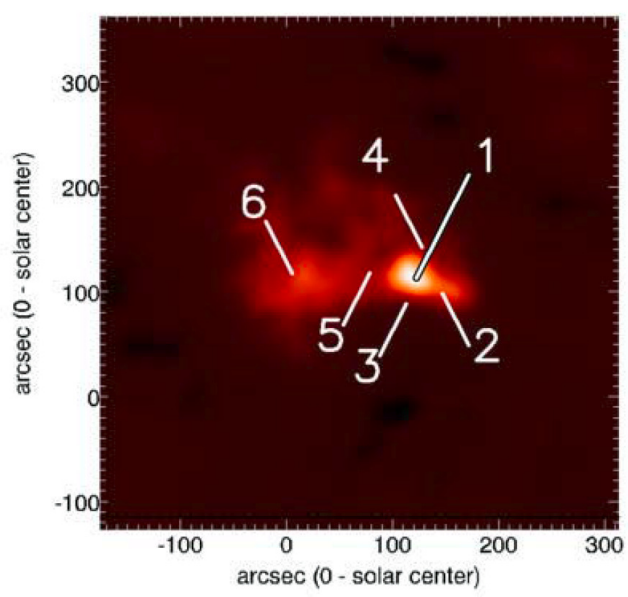

Рис. 1. Изображение AO NOAA 11283 по наблюдениям на радиогелиографе Nobeyaта 6 сентября 2011 г. на частоте 17 ГГц в интенсивности (параметр Стокса I). Указаны положения пикселей изображения АО, для которых построены временные профили радиоизлучения, приведенные на рис. 2
Для работы были использованы наблюдения Солнца, выполненные на NoRH на частоте 17 ГГц $(1.76 \mathrm{~cm})$ в интенсивности (параметр Стокса I). Мы провели исследование пространственного распределения КПК микроволнового излучения для двух АO: NOAA 11283 и 11302, наблюдавшихся 6 и 25 сентября 2011 г. соответственно.

В таблице 1 приведена информация о вспышках, которые произошли в рассмотренных АO в интервал времени наблюдений на NoRH, согласно бюллетеням Space Weather Prediction Center The Preliminary Report and Forecast (SWPC PRF) № 1880 и $1882^{1}$. Для нашей работы существенно, что перед вспышкой M5.3 6 сентября и вспышкой M4.4 25 сентября не было других вспышек c момента начала наблюдений на NoRH, что позволяет использовать эти интервалы для выявления КПК.

Для анализа КПК в АO NOAA 11283 использовались готовые изображения в fits-формате с шагом 3 мин $^{2}$. Для анализа KПК в АO NOAA 11302 был выполнен синтез радиоизображений в нестандартном режиме с шагом по времени 1 мин и временем усреднения 10 сек по алгоритмy Koshix. Далее для обеих AO на всех изображениях полного диска Солнца выделялась исследуемая AO (fieldof-view - FOV) с учетом вращения Солнца и строились временные профили яркостных температур в 6 выбранных пикселях изображения АО.

Таблица 1. Данные о вспышках 6 и 25 сентября 2011 г. в интервале времени наблюдений на NoRH по данным бюллетеней SWPC PRF № 1880 и 1882. В колонке "Старт" приведены моменты начала вспышек, "Пик" - моменты максимума вспышек, "Стоп" - моменты окончания вспышек, GOES - мощность вспышек согласно классификации GOES

\begin{tabular}{cccccc}
\hline NOAA & Дата & Старт & Пик & Стоп & GOES \\
\hline 11283 & 6 сентября 2011 & $01: 35$ & $01: 50$ & $02: 05$ & M5.3 \\
11302 & 25 сентября 2011 & $02: 27$ & $02: 33$ & $02: 37$ & M4.4 \\
11302 & 25 сентября 2011 & $03: 27$ & $03: 32$ & $03: 38$ & C7.9 \\
11302 & 25 сентября 2011 & $04: 31$ & $04: 50$ & $05: 05$ & M7.4 \\
\hline
\end{tabular}

На рис. 1 приведен фрагмент радиоизображения Солнца на частоте 17 ГГц, содержащий АО NOAA 11283, на котором указаны положения пикселей, для которых построены временные профили яркостных температур. Аналогичный фрагмент для АO NOAA 11302 показан на рис. 3. Соответствующие временные профили яркостных температур на частоте 17 ГГц представлены на рис. 2 и 4. Рассмотрим их подробнее.

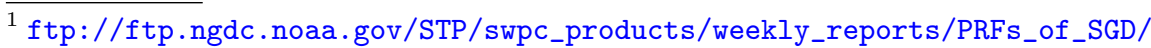

2 https://solar.nro.nao.ac.jp/norh/fits/3min/
} 
2.2 Предвспышечные временные профили микроволнового излучения АO NOAA 11283
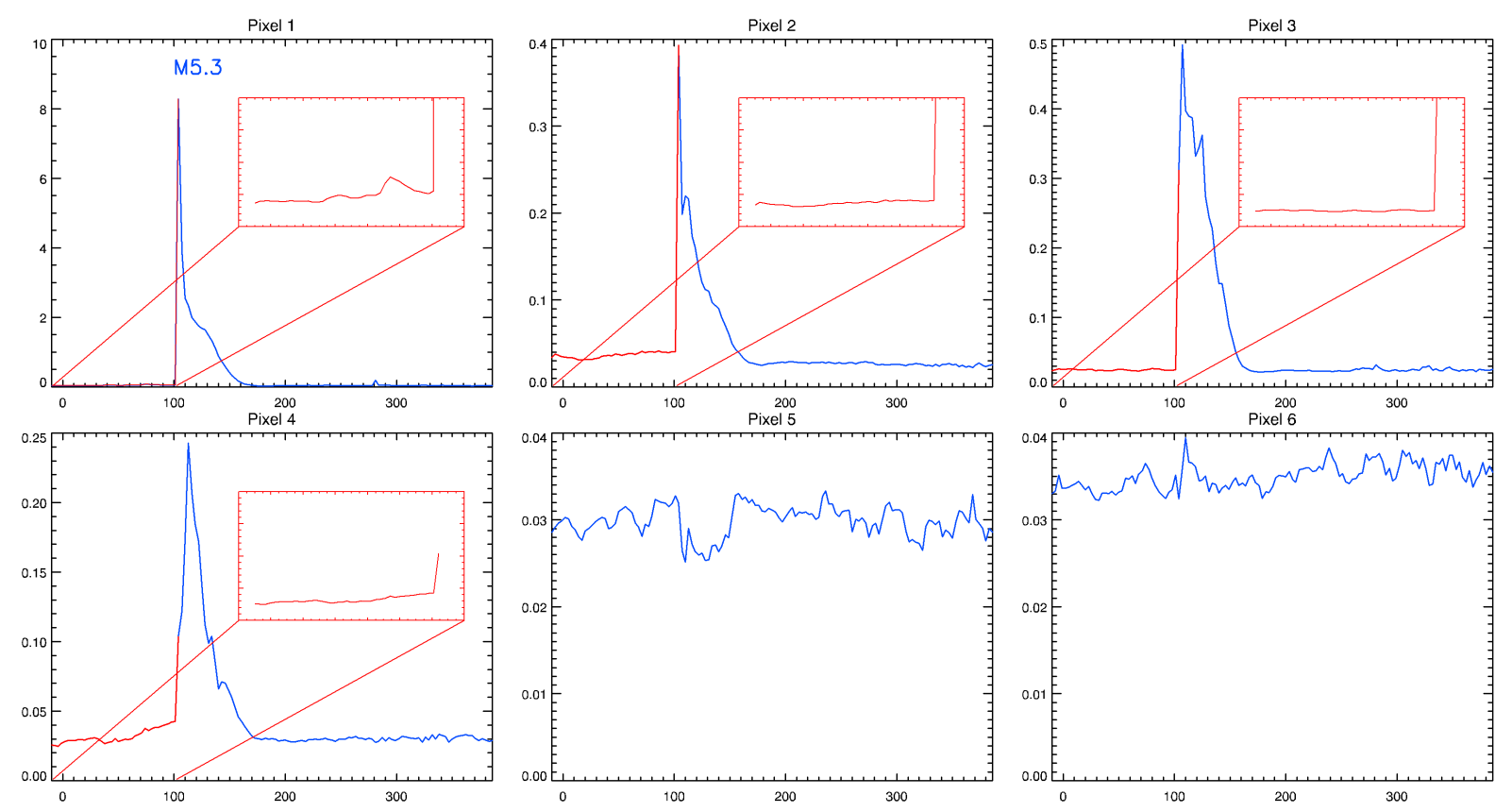

Рис. 2. Временные профили микроволнового излучения различных участков AO NOAA 11283 (положения пикселей указаны на рис. 1) на частоте 17 ГГц в интенсивности (параметр Стокса I) по наблюдениям на радиогелиографе Nobeyama 6 сентября 2011 г. Красным цветом на временных профилях 1-4 показаны предвспышечные участки. Предвспышечные фрагменты временных профилей показаны в более крупном масштабе на красных вставках. На всех красных вставках масштаб одинаковый. На всех панелях по оси абсцисс - время в минутах с начала суток UT (0 соответствует 00:00 UT), по оси ординат - яркостная температура в MK

На рис. 2 на четырех панелях красным цветом выделены фрагменты временных профилей с момента начала наблюдений до момента начала вспышки М5.3. Эти же фрагменты показаны отдельно на красных вставках в одинаковом масштабе.

На рис. 2 видно, что в пикселе 5 , соответствующем межпятенной зоне, и в пикселе 6 , находящемся в хвостовом пятне, нет никаких изменений в характере временных профилей в течение всего дня наблюдений. Пиксель 1 находится в головном пятне и области, в которой наблюдалась максимальная яркостная температура во время вспышки. Именно в этом пикселе проявляется предвспышечный цуг длительностью примерно 60 мин, состоящий из 3 циклов колебаний. Следует отметить, что во всех ранее обнаруженных предвспышечных цугах с совершенно разными длительностями (от нескольких минут до часа и более) число циклов колебаний было не более 10 и составляло в среднем 5 (Abramov-Maximov, Bakunina, 2020).

Пиксели 2, 3 и 4 соответствуют периферии головного пятна. Вспышка в микроволновом диапазоне в них наблюдается, но на порядок слабее, чем в пикселе 1. В пикселе 3 можно заметить лишь слабый намек на KПК, в пикселе 2 и 4 KПК не видны.

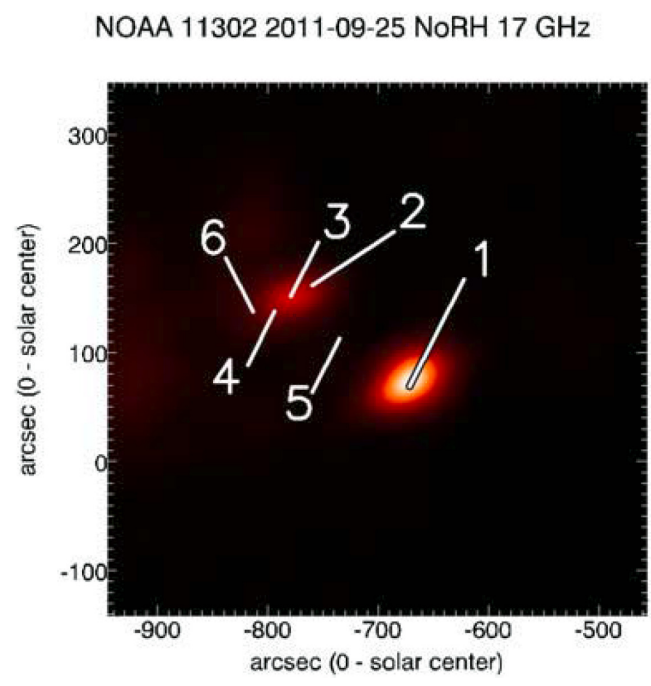

Рис. 3. Изображение AO NOAA 11302 по наблюдениям на радиогелиографе Nobeyama 25 сентября 2011 г. на частоте 17 ГГц в интенсивности (параметр Стокса I). Указаны положения пикселей изображения АО, для которых построены временные профили радиоизлучения, приведенные на рис. 4 
Таким образом, в АO NOAA 112836 сентября 2011 г. наблюдается предвспышечный цуг КПК яркостной температуры микроволнового излучения, который генерируется в той же самой компактной зоне над головным пятном АО, в которой была максимальная яркостная температура во время вспышки.

\section{3 Предвспышечные временные профили микроволнового излучения АO NOAA 11302}
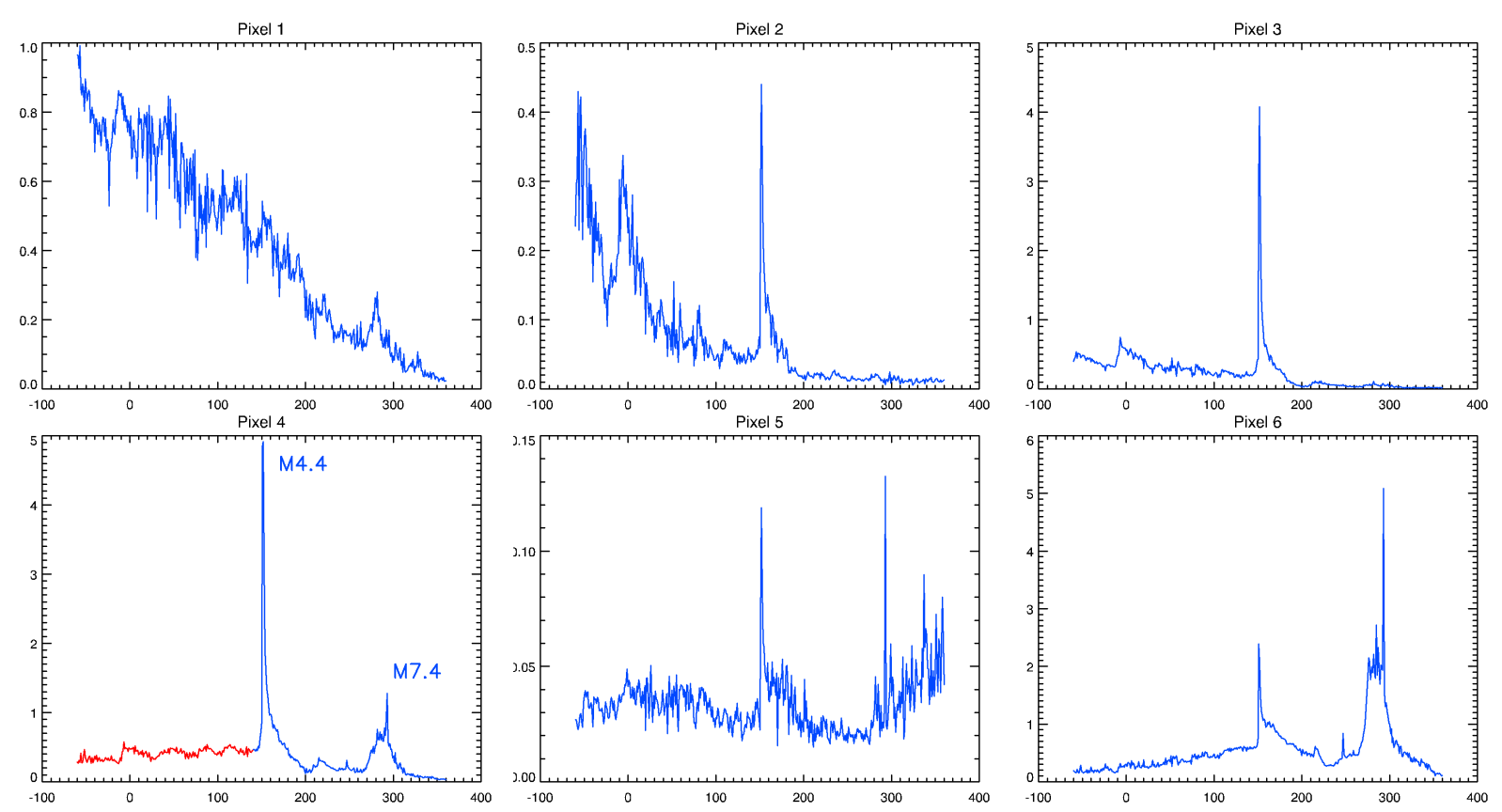

Рис. 4. Временные профили микроволнового излучения различных участков АO NOAA 11302 (положения пикселей указаны на рис. 3) на частоте 17 ГГц в интенсивности (параметр Стокса I) по наблюдениям на радиогелиографе Nobeyama 25 сентября 2011 г. Красным цветом на временном профиле для пикселя 4 показан предвспышечный цуг. На всех панелях по оси абсцисс - время в минутах с начала суток UT (0 соответствует 00:00 UT), по оси ординат - яркостная температура в MK

На рис. 4 видно, что в пикселе 1 , соответствующем головному пятну, ни вспышка, ни колебания не проявляются. В пикселях $2,3,5$ и 6 вспышка видна в той или иной степени, но заметных колебаний не видно. Наиболее сильно предвспышечный цуг, состоящий из 4 циклов колебаний, наблюдается только в пикселе 4 , находящемся в хвостовом пятне. Длительность цуга составляет примерно 2 часа, длительность циклов колебаний изменяется от 40 до 20 минут. Следует отметить, что именно в пикселе 4 наблюдается наибольшая яркостная температура во время вспышки. Возможно, слабые KПК можно выделить в пикселе 3 , находящемся рядом с пикселем 4 , и еще более слабые в пикселе 2 , также расположенном в хвостовом пятне $\mathrm{AO}$, но на большем удалении от пикселя 4 , чем пиксель 3 .

Таким образом, в АО 11302 за 2 часа до вспышки класса М4.4 наблюдаются КПК микроволнового излучения, локализованные в компактной зоне, в которой была наибольшая яркостная температура во время вспышки.

\section{3 Выводы}

По наблюдениям на радиогелиографе Nobeyama мы проанализировали временные профили яркостных температур микроволнового излучения на частоте 17 ГГц двух АО (NOAA 11283 и 11302) перед вспышками М5.3 и М4.4 соответственно. В обоих случаях наблюдаются цуги предвспышечных КПК с невысокой добротностью (длительностью $3-4$ цикла колебаний). КПК микроволнового излучения локализованы в компактных зонах, совпадающих с зонами, в которых наблюдалась максимальная яркостная температура во время вспышек.

Работа выполнена в рамках Государственного задания (тема 1021032422589-5). 


\section{Литература}

Абрамов-Максимов В.Е., Бакунина И.А., 2018. Ядерная физика. Т. 81. № 3. С. 366. [AbramovMaximov V.E., Bakunina I.A., 2018. Phys. At. Nuclei, vol. 81, no. 3, pp. 379-383.]

Авдюшин С.В., Богомолов А.Ф., Борисова Е.А. и др., 1985. Доклады АН СССР. Т. 283. № 1. С. 67. [Avdyushin S.I., Bogomolov A.F., Borisova E.A., et al., 1985. Akademiia Nauk SSSR, Doklady, vol. 283, no. 1, pp. 67-70. (In Russ.)]

Аверьянихина Е.А., Паупере М., Озолинс Г., Элиас М., 1982. Иссл. Солнца и красных звезд. Рига: Зинатне. №. 16. С. 61. [Aver'yanikhina E., Paupere M., Ozolins G., Elias M., 1982. Issled. Solntsa i Krasnykh Zvezd., no. 16, pp. 61-74. (In Russ.)]

Алешин В.И., Кобрин М.М., Коршунов А.И., 1973. Изв. ВУЗов. Радиофизика. Т. 16. С. 747. [Aleshin V.I., Kobrin M.M., Korshunov A.I., 1973. Radiophys. Quantum Electron., vol. 16, no. 5, pp. 571-576.]

Арбузов С.И., 1979. Изв. ВУЗов. Радиофизика. Т. 22. С. 1165. [Arbuzov S.I., 1979. Radiophys. Quantum Electron., vol. 22, no. 10, pp. 803-811.]

Берулис И.И., Молчанов А.П., Олянюк В.П. и др., 1973. Изв. ВУЗов. Радиофизика. Т. 16. С. 1362. [Berulis I.I., Molchanov A.P., Olyanyuk V.P., et al., 1973. Radiophys. Quantum Electron., vol. 16, no. 9, pp. 1047-1049.]

Берулис И.И., Козловский А.Л., Лосовский В.Я. и др., 1983. Астрон. журн. Т. 60. С. 974. [Berulis I.I., Kozlovskii A.L., Losovskii B.Y., et al., 1983. Sov. Astron., vol. 27, no. 5, p. 563.]

Быстров М.В., Кобрин М.М., Снегирев С.Д., 1978. Письма в Астрон. журн. Т. 4. С. 143. [Bystrov M.V., Kobrin M.M., Snegirev S.D., 1978. Sov. Astron. Lett., vol. 4, pp. 76-77.]

Быстров М.В., Кобрин М.М., Снегирев С.Д., 1979. Геомагнетизм и аэрономия. Т. 19. С. 306. [Bystrov M.V., Kobrin M.M., Snegirev S.D., 1979. Geomagn. Aeron., vol. 19, pp. 197-199.]

Кобрин М.М., Пахомов В.В., Дурасова М.С. и др., 1973а. Изв. ВУЗов. Радиофизика. Т. 16. С. 1350. [Kobrin M.M., Pakhomov V.V., Durasova M.S., et al., 1973a. Radiophys. Quantum Electron., vol. 16, pp. 1036-1039.]

Кобрин М.М., Коршунов А.И., Снегирев С.Д., Тимофеев Б.В., 1973b. Солнечные данные. № 10. C. 79. [Kobrin M.M., Korshunov A.I., Snegirev S.D., Timofeev B.V., 1973b. Bull. Solnechnye Dannye, no. 10, p. 79-85. (In Russ.)]

Кобрин М.М., Семенова С.В., Пахомов В.В., Пахомова О.А., Фридман В.М., 1981. Астрон. циркуляр. № 1201. C. 1. [Kobrin M.M., Semenova S.V., Pahomov V.V., Pahomova O.A., Fridman V.M., 1981. Astron. Tsirk., no. 1201, p. 1.]

Коршунов А.И., Прокофьева Н.А., 1976. Солнечные данные. № 2. С. 52. [Korshunov A.I., Prokof'eva N.A., 1976. Bull. Solnechnye Dannye, no. 2, pp. 52-56. (In Russ.)]

Abramov-Maximov V.E., Gelfreikh G.B., Shibasaki K., 2011. Solar Phys., vol. 273, pp. 403-412.

Abramov-Maximov V.E., Bakunina I.A., 2019. Geomagn. Aeron., vol. 59, no. 7, pp. 822-826.

Abramov-Maximov V.E., Bakunina I.A., 2020. Geomagn. Aeron., vol. 60, no. 7, pp. 846-852.

Durasova M.S., Kobrin M.M., Yudin O.I., 1971. Nature, vol. 229, pp. 82-84.

Gelfreikh G.B., Grechnev V.V., Kosugi T., Shibasaki K., 1999. Solar Phys., vol. 185, pp. $177-191$.

Gelfreikh G.B., Nagovitsyn Yu.A., Nagovitsyna E.Yu., 2006. Publ. Astron. Soc. Japan, vol. 58, pp. 29-35.

Sych R., Nakariakov V.M., Karlicky M., Anfinogentov S., 2009. Astron. Astrophys., vol. 505, pp. 791-799.

Tan B., Yu Z, Huang J, Tan C, Zhang Y., 2016. Astrophys. J., vol. 833, id. 206. 


\title{
Precursors of solar flares in the microwave range
}

\author{
V.E. Abramov-Maximov ${ }^{1}$, I.A. Bakunina ${ }^{2}$ \\ ${ }^{1}$ Central (Pulkovo) Astronomical Observatory of RAS, Pulkovskoye sh. 65/1, St. Petersburg 196140, Russia \\ beam@gaoran.ru \\ 2 HSE University, Bolshaya Pecherskaya 25/12, Nizhny Novgorod 603155, Russia
}

\begin{abstract}
We present a study of the spatial distribution of quasiperiodic oscillations of microwave emission in two active regions before M-class flares. We have studied two events: NOAA 11283 on September 6 and NOAA 11302 on September 25, 2011. We used the Nobeyama Radioheliograph (NoRH) daily observations at a frequency of $17 \mathrm{GHz}$. In both cases we found the preflare wave trains of brightness temperature oscillations of microwave emission in active regions. The duration of wave trains is about 3-4 pulses. We found that the source of oscillations was a compact zone in the active region, which coincides with the place of maximum brightness during a flare.
\end{abstract}

Key words: Sun, flares, radio emission, quasiperiodic oscillations 